PRAXIS

ecucativa

Universidad Nacional de La Pampa

Facultad de Ciencias Humanas

Instituto de Ciencias de la Educación

para la investigación interdisciplinaria

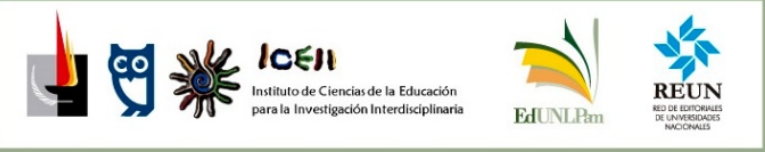

ISSN 2313-934X

SANTA ROSA, LA PAMPA, ARGENTINA

Correo electrónico: iceii@humanas.unlpam.edu.ar

Disponible en https://cerac.unlpam.edu.ar/index.php/praxis

Las clases de economía en la escuela secundaria durante la emergencia sanitaria por COVID-19. Artículo de Jorge Damián Lo Cascio. Praxis educativa, Vol. 26, No 1 enero - abril 2022. E - ISSN 2313-934X. pp. 1-17. https://dx.doi.org/10.19137/praxiseducativa-2022-260118

\title{
Las clases de economía en la escuela secundaria durante la emergencia sanitaria por COVID-19
}

Economics classes in high school during the health emergency by COVID-19

Aulas de economia no ensino médio da Argentina durante a emergência de saúde COVID19

\section{Jorge Damián Lo Cascio}

Universidad de Buenos Aires, Argentina

jorge.locascio@gmail.com

ORCID 0000-0002-8135-7495

Recibido: 2021-06-02 | Revisado: 2021-03-17 | Aceptado: 2021-10-27 


\section{Resumen}

Este artículo tiene como objetivo describir las clases de economía en las escuelas secundarias de la Ciudad de Buenos Aires (Argentina) durante la emergencia sanitaria por el COVID-19. La particular situación de clases sin presencialidad implicó que las escuelas continuaran funcionando, garantizando la continuidad pedagógica de sus estudiantes, pero dando lugar a modificaciones de la vida escolar que impactaron en las configuraciones de las clases.

Para la recolección de datos, se utilizó, en primer lugar, una encuesta autoadministrada mediante un formulario en línea y, luego, se realizaron entrevistas semiestructuradas realizadas por videollamada. Los resultados muestran tres configuraciones de clases de acuerdo con los espacios, tiempos, frecuencia, agrupamientos de estudiantes y medios de comunicación utilizados. El primer grupo se caracteriza por el intento de emular las clases con presencialidad. Un segundo grupo presenta rasgos donde la clase aparece diferenciada o personalizada en términos de espacios, tiempos, frecuencias, y medios utilizados. Un tercer grupo aparece identificado con clases en tiempo asincrónico, con espacios difusos o no delimitados de clase.

Palabras claves: clases, enseñanza, escuela secundaria, aulas, técnicas de observación en el aula

\section{Abstract}

This article describes economics classes in secondary schools in Argentina during the health emergency by COVID-19. The situation of off- site classes meant that the schools continued to function, ensuring the pedagogical continuity of their students, but resulting in changes in school life that impacted the configurations of the classes.

For data collection, a self-administered online survey form and semi-structured interviews conducted by video call were conducted.

The results show three large class configurations according to the spaces, times, frequency, and media used. The first group is characterized by an attempt to emulate on-site classes. A second group presents traits where the class appears differentiated, customizing in the spaces, times, frequencies, and media used. A third group is identified with classes in asynchronous times, with diffuse or not delimitated class places.

Keywords: class organization; teaching experience; high schools; classrooms; classroom observation techniques.

\section{Resumo}

Este artigo tem como objetivo descrever as aulas de economia em escolas secundárias, na Argentina, durante a emergência de saúde pelo COVID-19. A situação particular das aulas sem presencial fez com que as escolas continuassem funcionando, garantindo a continuidade pedagógica de seus alunos, mas resultando em mudanças na vida escolar que impactaram as configurações das aulas. Uma pesquisa auto administrada usando um formulário online e, em seguida, entrevistas semiestruturadas realizadas por videochamadas foram primeiramente utilizadas para a coleta de dados.

Os resultados mostram três grandes configurações de classe de acordo com os espaços, horários, frequência e mídia utilizadas. 0 primeiro grupo é caracterizado por uma tentativa de emular classes com presencial. Um segundo grupo apresenta características onde a classe aparece diferenciada, personalizada nos espaços, horários, frequências e mídias utilizadas. Um terceiro grupo é identificado com aulas em tempos assíncronos, com vagas difusas e delimitadas.

Palavras-chave: organização de classes; experiência de ensino; escolas secundárias; salas de aula; técnicas de observação em sala de aula 


\section{Introducción}

La excepcionalidad del momento histórico que atraviesa el mundo y, en especial, la escuela sobresale y desborda cualquier descripción, pero vale la pena, como afirma Arata, "hacer archivo de esta experiencia" (2020, p. 69). En un sentido similar, Dussel sostiene que "será necesario, en el futuro, documentar mejor qué representó la continuidad pedagógica en las escuelas" (2020, p. 343).

En tal sentido, este artículo describe, a partir de testimonios docentes, las estructuras y organización de las clases sin presencialidad en la Ciudad de Buenos Aires durante el contexto de aislamiento social, preventivo y obligatorio (ASPO) dispuesto por el gobierno argentino el 20 de marzo de 2020 en respuesta al comienzo de la pandemia por el COVID-19. Esta situación no impidió que las escuelas continuaran funcionando, dando lugar a procesos de continuidad pedagógica sin presencialidad. Así, ocurrieron múltiples y heterogéneas modificaciones de la vida escolar por las restricciones de presencialidad que impactaron tanto en las clases como en las prácticas de enseñanza o las experiencias y aprendizajes del estudiantado.

Como afirman Sadovsky y Castorina (2020), la expresión "continuidad pedagógica" que propuso el Ministerio de Educación en Argentina, desde el comienzo de la emergencia sanitaria, tuvo gran adhesión en la comunidad educativa, pero recayó en el personal docente la principal responsabilidad de garantizar la continuidad, con mucho esfuerzo y poniendo a disposición recursos de todo tipo, en especial muchas horas de trabajo.

Ahora bien, siguiendo a los autores citados, el encuentro en las aulas es (¿fue?) vital para organizar las situaciones didácticas y las interacciones docentes se configuran en las clases presenciales. Esas escuelas "de carne y hueso", como las referencia Terigi (2020), son diversas, pero contienen elementos comunes en tanto institución social. Un elemento central, marca la autora, es el contexto específico de aprendizaje, un entorno social especializado que se ha consolidado a lo largo del tiempo y ha adquirido cierta regularidad y estabilidad en la organización del trabajo educativo. Por tanto, Terigi (2020) afirma que la escuela produce formas específicas de aprendizaje en los estudiantes, pero "no se trata de 'el aprendizaje y su contexto', como si el aprendizaje pudiera definirse con independencia del contexto en que se realiza y, al hablar del contexto escolar, solo nos quedara por ver cómo este influye sobre el aprendizaje" (p. 244).

Entonces, el confinamiento de la población por la emergencia sanitaria reconfiguró el contexto escolar, por tanto, modificó tanto los aprendizajes como las prácticas docentes y, en consecuencia, las clases. En un sentido similar lo plantean Barberis y Toibero (2020) cuando afirman que "la idea de aula conocida como estructura material-comunicacional desapareció como espacio de encuentro y construcción de saberes, generando así la necesidad de construir 'nuevas aulas'"' (p. 201).

Ahora bien, conviene pensar las clases ("nuevas aulas") con los aportes teóricos de Davini (2015) respecto a las prácticas docentes. La autora define las prácticas docentes como las capacidades de la acción profesional en la escuela, lo cual excede la actuación en el aula. Las 
capacidades a las cuales refiere se pueden agrupar en dos esferas, la primera denominada "organización de la enseñanza", que alude a la programación, lo cual implica definir propósitos de enseñanza y objetivos de aprendizaje; organizar y secuenciar los contenidos; disponer una metodológica de enseñanza; planificar actividades de aprendizaje y las tareas del estudiantado; y seleccionar materiales y recursos para llevar adelante las actividades. La segunda esfera describe "las decisiones docentes en la acción" que implica el momento donde la programación se concretiza en un ambiente con sujetos reales que interactúan y, por tanto, pueden modificar el desarrollo de la programación. En consecuencia, las decisiones docentes en la acción refieren al momento áulico, al desarrollo de la clase como lugar y tiempo definido para la enseñanza en su singularidad.

De este modo, es preciso rescatar algunas de las dimensiones planteadas. En primer lugar, el manejo del espacio, dado que la autora concibe a la clase como "el ambiente interno y comunicativo que vincula a alumnos, docentes y recursos de aprendizaje, enmarcados en coordenadas espaciales, temporales y socioculturales" (Davini, 2015, p. 91). En dicho ambiente, cada docente debe decidir sobre la distribución de lugares-cosas-actividades estableciendo la posibilidad de "circulación" o flujos de intercambio en la interacción con otros. En la situación de emergencia sanitaria por la pandemia de COVID-19, surge una nueva dimensión por las restricciones a la presencialidad escolar: los medios de comunicación utilizados para establecer la circulación y flujos de intercambios.

En estrecha vinculación con las dimensiones anteriores, aparece la gestión del tiempo. En el planteo de Davini (2015), se asume que la enseñanza implica una secuencia de tareas que se desarrollan, necesariamente, en el tiempo, al igual que los aprendizajes, pero ambos tiempos son diferentes. La enseñanza se realiza en un tiempo externo, medible, con desarrollo lineal y uniforme, regulado por la institución y orientado siempre al futuro, sin vuelta atrás. En tanto, los aprendizajes tienen tiempos internos y variables, con posibles retrocesos productivos. De este modo, y dado el objetivo de este trabajo y la metodología utilizada para la recolección de datos, que coloca en el centro la mirada docente, será posible considerar solamente la gestión del tiempo de enseñanza. En consecuencia, la gestión del tiempo se vincula, en primer lugar, con la jerarquía, en tanto importancia diferencial, del contenido que se enseña, su complejidad y valor educativo. En segundo lugar, con algún criterio de regulación, ritmo y frecuencias de las tareas para el logro del aprendizaje. En último lugar, algún criterio refiere a la secuencia integradora, es decir, entender a las tareas no como unidades sueltas, sino en tanto hilos de continuidad de un proceso integrado donde las tareas se complementan.

Así, la cuarta dimensión refiere a la coordinación del grupo dado que considera a la clase como una situación grupal y, por tanto, el aprendizaje de cada estudiante se produce con la mediación del grupo (Davini, 2015). De allí la importancia de la comprensión de las formas de agrupamiento y su coordinación, mediante actividades y tareas, por parte del equipo docente.

A partir de los datos recolectados tanto con las encuestas autoadministradas como con las entrevistas realizadas, es posible describir las clases sin presencialidad considerando las 
dimensiones propuestas: los tiempos, los espacios, la frecuencia, los agrupamientos de estudiantes y los medios de comunicación utilizados. De este modo, se recabaron indicios que permitían reconocer tres tipos de estructuras y organización de las clases sin presencialidad.

El próximo apartado presenta los principales aspectos metodológicos de la investigación que refieren a los instrumentos para la recolección de datos y construcción de la muestra. En tanto, en el tercer apartado, se describen las estructuras y organizaciones de las clases de economía en las escuelas secundarias de la Ciudad de Buenos Aires durante el ASPO decretado por la pandemia de COVID-19. En la última sección, se delinean algunas conclusiones.

\section{Metodología}

Este apartado detalla los instrumentos para la recolección de datos y la construcción de la muestra. Vale resaltar que los resultados que se describen en este artículo son derivados de una investigación más amplia para la tesis de maestría en Educación de la Universidad de Quilmes, que busca describir las prácticas de enseñanza de economía en las escuelas secundarias de la Ciudad de Buenos Aires. ${ }^{i}$

Se utilizan dos instrumentos para la recolección de datos sobre las variables que permiten describir la estructura y organización de las clases sin presencialidad en el contexto de la emergencia sanitaria por COVID-19. El primero de los instrumentos es una encuesta autoadministrada mediante un formulario en línea que busca recabar información para conocer datos personales, laborales y de formación académica del equipo docente. Se utilizó un cuestionario que abarcaba preguntas abiertas y cerradas. Se realizó una prueba piloto con colegas cercanos que permitió mejorar las preguntas tanto en sus formas como en su contenido, en especial, se realizó una revisión respecto a la cuestión de género y el uso de lenguaje inclusivo.

La difusión del formulario se realizó mediante grupos de docentes de economía tanto por vía correo electrónico como mediante redes sociales. Esta situación provocó la respuesta de más de setenta docentes de todo el país, por tanto, la selección de casos consideró no solo el desempeño como docente de economía en la Ciudad de Buenos Aires, sino también género, antigüedad, tipo de gestión del establecimiento y turno de desempeño.

De esta forma, las respuestas dieron la posibilidad de armar un provisorio, pero importante, perfil de los docentes de economía que posibilitó la selección criteriosa para componer una muestra balanceada para la implementación del segundo instrumento de recolección de datos: las entrevistas semiestructuradas.

De este modo, se seleccionaron, para la muestra, nueve docentes, de las cuales siete son mujeres y dos son varones. En cuanto a la distribución por rangos etarios, la muestra contiene una docente de entre 30 y 40 años, cinco docentes entre 40 y 50 años y una mayor a 50 años. Respecto al tipo de gestión, hay cinco docentes que trabajan en escuelas de gestión pública, tres de gestión privada y una de escuelas secundarias pertenecientes a la Universidad de Buenos Aires. En relación con las titulaciones, cuatro tienen título de "profesor/a de economía, Ciencias Económicas u otro similar", dos son contadoras públicas, una es "licenciado/a en Administración u 
otro similar", un "licenciado/a en economía u otro similar" y, finalmente, una posee el título de técnica superior en Administración de Empresas.

Tanto en la formulación de la encuesta como de las preguntas básicas del guion de la entrevista, se tomó como referencia el trabajo de Cols (2007). Así, para preparar y efectuar las entrevistas, se utilizó la información proveniente de la encuesta para ajustar el énfasis de cada una de las preguntas de modo de mejorar la eficacia del instrumento. La realización de las entrevistas se llevó a cabo mediante videollamada, su duración promedio fue de 50 minutos y se buscó recoger expresiones del personal docente que permitan describir su práctica de enseñanza por medio de un lenguaje cercano a la cotidianeidad del trabajo docente. Para ello, se efectuó una entrevista exploratoria con un docente de secundaria, pero de otro espacio curricular.

Por su parte, la técnica de análisis de datos utilizada siguió el esquema propuesto por la teoría fundamentada (Glaser y Strauss, 1967; Soneira, 2006). De esa forma, es posible identificar cuatro etapas básicas. La primera consistió en la transcripción y el ordenamiento de los materiales de campo. Así, se procedió tanto a reordenar la información recolectada con las encuestas como a desgrabar las nueve entrevistas realizadas. También se pasaron en limpio las anotaciones efectuadas durante las entrevistas. En segundo término, a partir de las categorías iniciales, se realizó una codificación abierta de los materiales de campo, lo cual permitió emparentar las conceptualizaciones y definiciones con los relatos a través de ejemplos o ilustraciones que surgen de segmentos de las entrevistas. Luego, en la codificación axial, se agruparon las categorías con base en los patrones encontrados, con los datos obtenidos en el trabajo de campo. En cuarto lugar, se buscó construir nuevas categorías descriptivas para las unidades seleccionadas en la investigación, es aquí donde surge la importancia de la estructura y organización de las clases sin presencialidad para el estudio de las prácticas de enseñanza de profesores de Economía en las escuelas secundarias de la Ciudad de Buenos Aires, objetivo de la tesis de maestría para la cual se desarrolló la investigación.

A continuación, se describen los resultados en relación con la estructura y organización de las clases sin presencialidad a partir de testimonios docentes.

\section{Resultados}

La emergencia sanitaria y la particular situación de confinamiento de la población no impidieron que las escuelas continuaran funcionando, pero sin presencialidad. Así, ocurrieron múltiples y heterogéneas modificaciones de la vida escolar por las restricciones de presencialidad que impactaron tanto en las clases, en las prácticas de enseñanza, como en las experiencias y aprendizajes de los estudiantes (Terigi, 2020).

Este artículo describe los resultados en relación con las estructuras y organización de las clases sin presencialidad a partir de testimonios docentes, es decir, desde un lugar de las prácticas docentes que, como afirman Basabe y Cols (2010), son una práctica social influenciada no solo por el contexto histórico, social y político, sino también por los rasgos de la institución escolar y la cultura institucional. 
A partir de encuestas y entrevistas, se describen las clases de economía en la escuela secundaria considerando los tiempos, los espacios, las frecuencias, las formas de agrupamientos de estudiantes y los medios de comunicación utilizados. Así, es posible hallar indicios que permiten reconocer tres tipos de las clases sin presencialidad, con características y elementos comunes para agrupar las experiencias recogidas en las entrevistas.

Un primer elemento que sale a la luz durante la particular situación de aulas sin presencialidad es la imposibilidad de observar, de forma directa, aquellos hechos triviales o afanes cotidianos a los cuales refería Jackson (1975) en su clásico La vida en las aulas. Esa vida escolar "naturalizada" en el mundo occidental a fuerza de repetición de rutinas, uniformidad de prácticas y obligatoriedad que hace posible reconocer el ambiente áulico como distintivo de cualquier otro ambiente. Esta vida escolar se vio conmovida por la restricción a la presencialidad y rompió el clásico ambiente de cualquier escuela secundaria, donde un agrupamiento de estudiantes a cargo de un/una profesor/a que enseña determinada disciplina en un lugar y tiempo definido.

Las escuelas tuvieron que improvisar una vida escolar, recuperar los elementos vitales para su funcionamiento sin contar con la presencialidad. Reconstruir la clase como lugar activo, como ambiente de interacciones personales entre docentes y estudiantes (Davini, 2015). Fue necesario recrear un espacio áulico, un tiempo de clase, una nueva forma de dialogo, poner en juego nuevas reglas y recursos para que la enseñanza ocurra o, al menos, tenga posibilidades de ocurrir.

\section{Estructura y organización de las clases sin presencialidad}

La influencia de la institución en la organización y estructura del dispositivo escolar durante la pandemia resulta imprescindible para describir las clases. ${ }^{\text {ii } A s i ́ ~ c o m o ~ l a ~ e s c u e l a, ~ e n ~}$ tanto institución social, afecta la enseñanza, también lo hace el establecimiento escolar. Cada escuela en tanto organización asume rasgos propios y formas distintivas de acuerdo con su contexto social y político; su entorno geográfico; la población que atiende; su dotación de docentes y definición de puestos de trabajo; su arquitectura, equipamiento y recursos (materiales y simbólicos). De esta manera, la cultural institucional influye en la enseñanza de variadas formas, pero sin desconocer rasgos comunes, repetibles y generalizables que hacen posible el estudio sistemático de la enseñanza (Contreras, 1990; Basabe y Cols, 2010).

Durante el ASPO, las clases se llevaron adelante sin presencialidad y cada escuela tuvo que lograr nuevas formas de trabajo para alcanzar sus variados, múltiples y diversos objetivos. De este modo, conviene rescatar algunos fragmentos de los relatos recogidos en las entrevistas y esbozar algunas conjeturas que refieren a la influencia de "lo institucional" en la estructura y organización de las clases de las/os docentes entrevistados.

La "bajada institucional", como la refieren las personas entrevistadas, formatea los límites, alcances y posibilidades de la clase y puede ser vista como una imposición que perjudica u obstruye la práctica docente, así lo manifiesta M.D. 
Yo estuve bastante agarrada con la dirección por la bajada de la asesora pedagógica y la secuencia que proponía... primero un audio que diga "En economía vamos a ver la escasez", luego tirar una imagen, otro audio de un minuto y mandarle una actividad con una foto y que hicieran la actividad... eso es la nada misma, eso es un desastre. (M.D., profesora, escuela pública vespertina)

En el mismo sentido lo expresa otra docente:

Se lo mandé por el grupo de WhatsApp porque al principio yo trabajaba mucho con ellos con el grupo de WhatsApp, pero después le puse Classroom, pero después la escuela decidió que todo tenía que ir por el blog institucional y ahí se empezó a complicar. Entonces tuve que dejar el blog (institucional) nada más y el grupo de WhatsApp. Las actividades se las mando por el blog y, si tienen alguna duda o alguien no puede acceder, me dice y vamos por el grupo de WhatsApp, pero al principio solo lo que intentaba era tratar de tener como una clase, entonces le mandaba como preguntas por semana para que me la respondan por WhatsApp y demás, pero después ya no, no se pudo hacer. (V.P. profesora, escuela pública matutina)

Es posible conjeturar que tanto V.P. como M.D., ante la disconformidad con la bajada institucional, buscan intersticios en los cuales organizar sus prácticas para compensar aquellos aspectos que, a su modo de ver, perjudican la enseñanza: "Esto es lo que, a nivel institucional, no estaría consensuado... es algo marginal, algo por mi cuenta" (M.D., profesora, escuela pública vespertina).

Por ejemplo, el uso del WhatsApp iii para comunicarse con los estudiantes de forma individual, poder asistirlos, explicarles, ayudarlos/as a realizar la tarea o contener emocionalmente a los y las estudiantes aparece como una herramienta por fuera de la regulación escolar, un medio, un espacio donde la institución no alcanza a imponerse. Las docentes encontraron este resquicio para llevar adelante aspectos o dimensiones de sus prácticas de enseñanza que consideran relevante.

Distinto es el caso de C.P. que relata la organización institucional con la naturalidad propia de quien no se siente perjudicada por las disposiciones de las autoridades:

Lleva un mes, porque es una actividad. Yo les mando la actividad con el soporte y ellos tienen una semana para contestarme, pero hasta el mes siguiente no le mando la próxima actividad. La escuela dijo de hacer así porque si no dice que se juntaban todas las actividades y no podían hacer, entonces así. Por ejemplo, yo ahora el lunes les mando una actividad y ellos tienen hasta el viernes para devolverme la actividad, pero igual se sigue recepcionando. Después, hasta fines de octubre, no vuelvo a mandar la siguiente actividad. (C.P., profesora, escuela pública matutina)

En otros pasajes de la entrevista, C.P. relata que, durante una actividad y otra, no hay comunicación con las y los estudiantes, salvo para reclamar la entrega mediante correo electrónico. La sensación que transmite C.P es de aceptación de la "bajada institucional" respecto a la frecuencia y organización de sus clases. 
Es llamativo que los y las docentes que no refieren a cuestiones institucionales, ni en forma positiva ni negativa, son aquellos y aquellas que tienen clases sincrónicas con una frecuencia similar al momento previo de la emergencia sanitaria. Es posible aventurar que el personal docente entrevistado siente, en estos casos, que la influencia institucional sobre sus prácticas de enseñanza es menor, dado que no rompe los tiempos de clases predefinidos para la presencialidad, no obstruye el proceso de comunicación o favorece, dadas las condiciones, un encuentro "lo más humano posible". En cierto sentido, la "bajada institucional" busca emular lo conocido.

Así las cosas, las definiciones institucionales posibilitan, obstruyen, favorecen, impiden, organizan las posibilidades del trabajo docente y de las tareas estudiantiles, al tiempo que disponen la distribución de los tiempos y los espacios donde ocurren las clases.

Un primer problema aparece respecto a la delimitación del ámbito escolar, por ser un espacio social especializado, separado del ámbito social más amplio y, por tanto, un tiempo y espacio delimitado y preciso (Basabe y Cols, 2010). Esta característica se pone, de cierta forma, en cuestión sin la presencialidad porque exige que cada institución educativa pueda recrear un espacio escolar, las clases, que sea capaz de delimitar y segmentar el tiempo escolar. La presencialidad garantiza, sin más, un espacio y un tiempo escolar.

A continuación, se identifican, a partir de los relatos docentes, elementos que permiten reconstruir la estructura y organización de las clases.

\section{Los espacios y tiempos de clases sin presencialidad}

Es posible agrupar a las clases ${ }^{i v}$ con los relatos del equipo docente en las entrevistas en tres grupos de acuerdo con el modo de agrupamiento de estudiantes, los espacios, tiempos, la frecuencia de contacto y los medios de comunicación.

Un primer grupo puede caracterizarse por el intento de emular la presencialidad, como se afirmó anteriormente: los y las docentes no manifestaron contrariedad ni asombro por la "bajada institucional". Así, estos relatos tienen en común la realización de clases semanales sincrónicas que duran entre 40 y 60 minutos, un tiempo y espacio escolar que reúne a docentes y estudiantes de forma virtual para interactuar utilizando diferentes plataformas de videollamadas. Esta propuesta cuenta con buena participación por parte del estudiantado: "Es buena, los chicos participan, también yo les grabo la clase porque a veces hay cuestiones que les quedan colgadas" (A.B., profesor, escuela privada matutina); "Es buenísima. Aparte lo hablaba con la secretaria de estudios de la escuela, hay muy poca gente que me debe trabajos, eso me pone contenta" (M.M., profesora, escuela privada matutina):

Vienen siendo cada quince días y ahora están siendo semanales porque hay algunos que se conectan... hay semanas que se conectan algunos, otras semanas con otros, entonces, más que nada, si alguno no pudo o estuvo con algún problema... [la participación es] poquito más de la mitad. (C.L., profesora, escuela pública matutina) 
Otra coincidencia en los relatos respecto a la clase sincrónica es la segmentación del tiempo interno en ese espacio. Suelen combinarse tres momentos en distinto orden: un segmento dedicado a la exposición o explicación docente, otro momento de intercambio, debate o puesta en común de las tareas resueltas y, finalmente, la presentación de una nueva tarea escolar. Dentro este último segmento, se habilita un dialogo para resolver dudas, preguntas o consultas relacionadas con las nuevas tareas escolares. Luego de la clase, la comunicación entre profesores/as y estudiantes, como la entrega de trabajos, se realiza mediante correo electrónico. "[En la clase] Primero hablamos, hablamos de la actividad y después tengo un Padlet donde les presento actividades y presento material teórico que le voy a armando a ellos" (C.L., profesora, escuela pública matutina); "Yo iba explicando, los iba dando los temas. También lo que les daba es una guía de preguntas orientadora" (E.A., profesora, escuela preuniversitaria UBA matutina);

Tengo clases con ellos por Zoom y Classroom, generalmente todos los martes tengo a veces 40 minutos o, si no alcanza el tiempo, es más. Un día antes, les comparto algún material, les comparto cuál va a ser ese recurso que voy a utilizar y después (en la clase) les explico, hago que ellos participen, trato de que sea dinámica. (A.B., profesor, escuela privada matutina) Yo con ellos tengo martes y viernes, el martes le subo el material, las actividades o lo que tengan que estar buscando. Los viernes hacemos el encuentro para sacar dudas sobre el tema, explicarlo y tratar de contárselos en los minutos que tenemos disponibles en el Zoom. (C.C., profesora, escuela privada matutina)

Empecé con ese tema sin decirles qué vamos a ver... vamos a ver un video y listo. Empezamos a debatir un poquito qué pasaba, qué significaba el billete y empezamos a debatir un poco. Les empecé a explicar qué función tenía el dinero, medio de pago. (M.M., profesora, escuela privada matutina)

De los relatos y los fragmentos seleccionados, se desprende que la clase sincrónica mediante plataformas de videollamada busca emular las clases presenciales: su organización y estructura es similar. En tal sentido, aparecen los elementos destacados por Jackson (1975) en relación con el espacio áulico, lugar privilegiado de interacciones personales dentro de la escuela. Hay un/una docente que presenta una propuesta de enseñanza destinada al conjunto de los/las estudiantes, regulando el flujo del diálogo y los recursos que se ponen en juego. Asimismo, se presenta la exigencia de cumplimiento de un horario escolar, un estar presente de forma virtual, un tiempo que el estudiante debe dedicarle a la escuela.

Un segundo grupo permite caracterizar clases que diferencian y personalizan los espacios, tiempos, frecuencias y medios de comunicación donde ocurre la enseñanza. Estos docentes incluyen, en un primer momento, la realización de clase sincrónica, pero, al contar con baja o nula participación, se ven obligados/as, más por sus concepciones como docentes que por "bajada institucional", a diferenciar la propuesta de enseñanza y, en consecuencia, las clases. En un relato, se afirma que:

Las alumnas no utilizan Zoom porque tienen los teléfonos saturados, así que terminé usando Meet, que lo tienen un poquitito más... Pero ahora tengo el 20\% del alumnado, 
con el resto, que no me puede seguir en la clase sincrónica, le mando trabajos y la recepción la hago por mail. (G.P., profesor, escuela pública nocturna) Luego, continúa:

Tengo preparada las dos metodologías, para los que se conectan a clase lo trabajo en clase $y$, al que no se puede conectar por diferentes problemas, porque no tiene conectividad, por el horario o porque la casa no da o por lo que fuese, le envío el trabajo por mail y después se lo recibo. (G.P., profesor, escuela pública nocturna)

Es posible pensar que, ante el fracaso de emular la presencialidad mediante las clases sincrónicas en plataformas de videollamadas, cada docente construye una clase asincrónica, lo cual refleja que se manejan tiempos, espacios, frecuencias y medios diferenciados, ajustados a las necesidades de la población estudiantil que asiste a esa escuela pública nocturna, ubicada en la Comuna 13 de la ciudad. Es posible conjeturar que la práctica de enseñanza de G.P. se centra en el aspecto relacional dejando en segundo plano los contenidos disciplinares, su relato presenta indicios en tal sentido cuando afirma: "no me dediqué a enseñar, me dediqué a acompañar, a contener, a escuchar".

El relato de G.P. no debe llamar la atención dado que, en el Informe preliminar de la encuesta a docentes del Ministerio de Educación de la Nación (2020), se afirma que el 98\% de los/as encuestados/as del nivel secundario afirmó que priorizó el vínculo con los/as estudiantes para acompañarlos/as en el aislamiento cuando se los consultó por las características de las tares y actividades de la propuesta de continuidad pedagógica.

Por su parte, más profunda es la diferenciación que realiza M.D., que llega al extremo de personalizar la enseñanza, es decir, ajustar los tiempos, espacios y frecuencias de clase de acuerdo con la disponibilidad y grado de avance del estudiantado. M.D. relata estar disponible para el momento en que sus estudiantes puedan consultarla, preguntarle o enviarle la actividad vía WhatsApp a su teléfono personal.

Se ha resuelto por el consejo consultivo, que se asignó a cada área, un día para trabajar por WhatsApp, entonces, ese día, tercero, cuarto y quinto año, el ciclo orientado, particularmente a nosotros, los viernes tenemos nuestras clases, todas juntas. (...) Pero el tema es que me parece que el medio que se eligió o se seleccionó no es el correcto porque no logramos la presencia de los chicos en esas "aulas virtuales". (M.D., profesora, escuela pública vespertina)

Ante la escasa participación de estudiantes en las clases sincrónicas por el grupo de WhatsApp que propone la "bajada institucional", M.D. asume una postura de personalizar la interacción con sus estudiantes de modo no solo de ajustarse a sus tiempos, sino también a sus intereses y problemáticas. En cierto modo, parece que M.D. busca cualquier tipo de excusa o situación para desarrollar su práctica de enseñanza, lo cual desdibuja el tiempo y espacio áulico, es decir, la clase aparece como un continuo. En un sentido similar lo entiende el trabajo de Roldán (2020) cuando afirma, en relación con las clases por WhatsApp, que "los tiempos no tienen demarcaciones claras; justamente, la esencia de la aplicación radica en la respuesta inmediata sin 
importar el día ni el horario en que se escribió el mensaje" (p. 29). "La mayoría se comunica de forma individual. La mayoría, no todos, pero es algo muy de la escuela y no solamente conmigo es la cosa personalizada" (M.D., profesora, escuela pública vespertina); "Me paso de estar corrigiendo unos trabajos mientras estaba cocinando porque te demandan en cualquier momento y yo trato de atenderlos... no dejo de ser docente, no importa cuándo llamen, ni la problemática que plantee, siempre se está orientando" (M.D., profesora, escuela pública vespertina);

Ayer empecé a trabajar a las nueve horas y terminé la última clase a la 19:45, que me aparece una alumna, que hoy le terminé de resolver sus temas... a las 20:30 horas y hasta las 22:30 horas con WhatsApp. No tengo límites. (M.D., profesora, escuela pública vespertina)

Finalmente, un tercer grupo refiere a una estructura de clase donde los tiempos y lugares de las prácticas de docentes tienen como característica saliente que no realiza clases sincrónicas. En consecuencia, los tiempos y lugares de clases son más difusos respecto a los grupos anteriores. En cuanto a la frecuencia en el envío de actividades, según los relatos obtenidos, se realiza en forma quincenal, en el caso de V.P.; en tanto, C.P. lo realiza una semana al mes ${ }^{v}$ de acuerdo con la "bajada institucional". La modalidad de clase asincrónica implica para cada docente enviar tareas para que los y las estudiantes resuelvan a partir de ciertos recursos que abarcan información relacionada con el contenido. Luego, el docente recibe las entregas de cada estudiante, generalmente mediante correo electrónico o WhatsApp. "La escuela tiene un blog donde sube las actividades, pero cada profesor se podía hacer su propio Classroom. Entonces, yo subo mis actividades al Classroom, donde se supone que ellos pueden entrar, ver preguntar y todo eso" (C.P., profesora, escuela pública matutina);

Yo estoy obligada a mandar la actividad por el blog de la escuela, entonces yo mando una actividad del blog de la escuela, ahí dejas las consignas y donde ellos tienen que reenviar la tarea y después tengo el grupo de WhatsApp. (V.P., profesora, escuela pública matutina)

En estas clases asincrónicas, el agrupamiento de estudiantes aparece como sección homogénea, una referencia a "todo el curso", pero es posible reconocer diferenciaciones, en el relato de V.P, asociadas a la ayuda o el acompañamiento para realizar las actividades. En el caso de C.P., no es necesaria la diferenciación dado el alto nivel de cumplimiento y el tipo de actividades.

\section{Conclusiones}

A partir de la decisión gubernamental de suspender el desarrollo de clases presenciales debido a la pandemia por COVID-19, desde el 16 de marzo de 2020, se comenzaron a poner en marcha, en todas las escuelas de Argentina, propuestas de continuidad pedagógica para garantizar la educación obligatoria. De esta forma, cada establecimiento educativo debió reorganizar la tarea docente de acuerdo con criterios diseñados al interior de cada escuela.

Partiendo del planteo de Basabe y Cols (2010), que sostienen que enseñar es actuar en la urgencia, decidir en la incertidumbre, en escenarios áulicos caracterizados por la inmediatez y 
multidimensionalidad, es posible pensar que el contexto de la emergencia sanitaria llevó al extremo estas condiciones. Así, las configuraciones de las estructuras de clases detectadas en los relatos de los y las docentes de Economía en escuelas secundarias de la Ciudad de Buenos Aires reflejan, en primer lugar, un arreglo institucional, es decir, una forma de anticipar, reducir la incertidumbre y recrear los espacios, tiempos, frecuencia y medios de comunicación para la enseñanza y los aprendizajes.

De esta forma, la enseñanza asumió, de forma radical, su naturaleza práctica, lo cual exigió reflexión y deliberación para tomar decisiones, tanto institucionales como docentes, que busquen resolver los múltiples problemas prácticos a los cuales se enfrentaron en la emergencia sanitaria. En consecuencia, durante la pandemia por COVID-19, la enseñanza, como toda acción social, se presentó, más que nunca, de forma singular, inédita, escapando a reglas preestablecidas. Así, el desafío teórico que asumió este trabajo fue dilucidar lo general y repetible a partir de los relatos del equipo docente para describir estructuras y organizaciones de clases ensayadas para afrontar lo que Dussel (2020) denomina "domiciliación" de la escuela.

El artículo asume el desafío de describir las estructuras de clases durante la emergencia sanitaria para "hacer archivo" de una experiencia inédita, evitando consideraciones valorativas y colocando las voces de los y las docentes en primera plana. Así las cosas, los resultados muestran tres configuraciones de clases de acuerdo con los agrupamientos de estudios, los espacios, los tiempos, la frecuencia, y los medios de comunicación utilizados.

El primer grupo se caracteriza por configurar sus clases como un intento de emular las clases con presencialidad. Estos relatos muestran la realización de clases semanales de forma sincrónica con una duración promedio de 60 minutos. De este modo, se estructura un tiempo y espacio escolar que reúne a docentes y estudiantes de forma regular para interactuar utilizando plataformas de videollamadas. Esta configuración de clase se sostiene porque cuenta con buena participación por parte del estudiantado. Un segundo grupo configura las clases de modo que se diferencian y personalizan los espacios, tiempos, frecuencias y medios de comunicación. Si bien, en un primer momento, estos/as docentes buscaron realizar sus clases de forma sincrónica, contaron con escasa participación estudiantil, lo cual los llevó a diferenciar la propuesta de enseñanza. Un tercer grupo refiere a una estructura de clase asincrónica, en consecuencia, los tiempos y lugares de clases son poco delimitados y más difusos respecto a los grupos anteriores. En cuanto a la frecuencia de clases, este grupo presenta menor regularidad ya que la modalidad de clase asincrónica implica enviar tareas para que los/as estudiantes las resuelvan y entreguen, generalmente, mediante correo electrónico o WhatsApp.

Ahora bien, los datos recolectados brindan indicios que permiten identificar la tensión marcada por Dussel en relación con las desigualdades sociales, principalmente "respecto a la conectividad digital, la comodidad del espacio de trabajo y las posibilidades de las familias de sostener y acompañar" (2020, p. 339). De este modo, es posible pensar que la escuela, cuando funciona en sede escolar, permite condiciones más igualitarias que en su funcionamiento en el ámbito doméstico. En tal sentido, si se compara la estructuración de clases que emulan la 
presencialidad con las configuraciones de clases asincrónicas, es posible encontrar indicios que reflejan las desiguales condiciones de acceso a dispositivos por parte de estudiantes, lo cual dificulta la posibilidad de establecer tiempos y espacios de clases delimitados con una frecuencia regular.

Finalmente, es preciso preguntarse si las tipologías descriptas en el artículo pueden reconocerse a nivel nacional para las escuelas secundarias. Por caso, sería posible utilizar las bases usuarias de la Encuesta a Docentes de escuelas de nivel secundario realizadas por el Ministerio de Educación en el marco de la Evaluación Nacional del Proceso de Continuidad Pedagógica. Estos datos de mayor alcance pueden arrojar evidencias de la correlación entre las configuraciones de las clases durante la emergencia sanitaria con características de la escuela y las condiciones sociales y económicas tanto de docentes como de estudiantes. De esta manera, será factible pensar con mayor profundidad en qué medida la no presencialidad es posible que ensanche la brecha en la experiencia escolar de los y las estudiantes, profundizando la segmentación del sistema educativo.

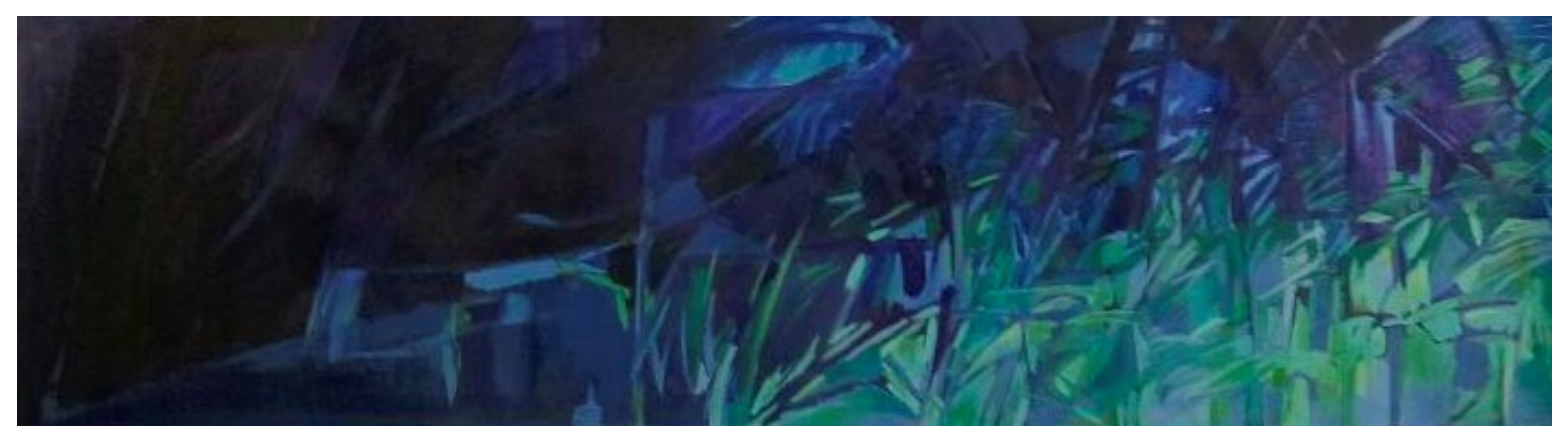

Nocturno, acrílico sobre papel. Paula Boh 


\section{Anexo}

\section{Cuadro 1}

Estructura de clases sin presencialidad

\begin{tabular}{|c|c|c|c|c|c|}
\hline \multicolumn{2}{|c|}{ Docente } & V.P. & C.P. & C.L. & M.M. \\
\hline \multicolumn{2}{|c|}{ Espacio curricular } & $\begin{array}{l}\text { Economía 3er año } \\
\text { Formación General }\end{array}$ & $\begin{array}{l}\text { Economía 3er año } \\
\text { Formación General }\end{array}$ & $\begin{array}{l}\text { Economía 3er año } \\
\text { Formación General }\end{array}$ & $\begin{array}{l}\text { Economía 3er año } \\
\text { Formación General }\end{array}$ \\
\hline \multicolumn{2}{|c|}{ Tipo de gestión } & Gestión pública & Gestión pública & Gestión pública & Gestión privada \\
\hline \multicolumn{2}{|c|}{ Turno } & Tarde & Mañana & Mañana & Mañana \\
\hline \multirow{5}{*}{ 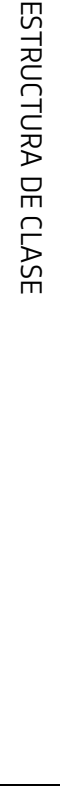 } & Agrupamiento & Todo el curso & Todo el curso & Todo el curso & Todo el curso \\
\hline & Espacios & No delimitado & No delimitado & $\begin{array}{l}\text { Aula } \\
\text { recreada/emulada en } \\
\text { forma virtual }\end{array}$ & $\begin{array}{l}\text { Aula } \\
\text { recreada/emulada en } \\
\text { forma virtual }\end{array}$ \\
\hline & Tiempo & Asincrónico & Asincrónico & $\begin{array}{l}\text { Clase sincrónica } \\
40 \text { a } 60 \text { minutos de } \\
\text { duración }\end{array}$ & $\begin{array}{l}\text { Clase sincrónica } \\
40 \text { minutos }\end{array}$ \\
\hline & Frecuencia & $\begin{array}{l}\text { Envío de actividades } \\
\text { con frecuencia } \\
\text { quincenal }\end{array}$ & $\begin{array}{l}\text { Se sube una } \\
\text { clase/actividad una } \\
\text { semana por mes }\end{array}$ & Semanal & Semanal \\
\hline & Comunicación & $\begin{array}{l}\text { Formal: blog de la } \\
\text { escuela } \\
\text { Informal: grupo de } \\
\text { WhatsApp y } \\
\text { mensajes privados } \\
\text { con estudiantes }\end{array}$ & $\begin{array}{l}\text { Blog de la escuela } \\
\text { Classroom } \\
\text { Correo electrónico } \\
\text { con estudiantes y } \\
\text { familias }\end{array}$ & $\begin{array}{l}\text { Plataforma } \\
\text { Reuniones virtuales } \\
\text { Correo electrónico } \\
\text { con estudiantes }\end{array}$ & $\begin{array}{l}\text { Plataforma } \\
\text { Reuniones virtuales } \\
\text { Correo electrónico } \\
\text { con estudiantes }\end{array}$ \\
\hline
\end{tabular}

\section{Cuadro 1}

Estructura de clases sin presencialidad (continuación)

\begin{tabular}{|c|c|c|c|c|c|c|}
\hline \multicolumn{2}{|c|}{ Docente } & C.C. & E.A. & A.B. & M.D. & G.P. \\
\hline \multicolumn{2}{|c|}{ Espacio curricular } & $\begin{array}{l}\text { Economía 4to } \\
\text { año Formación } \\
\text { Orientada }\end{array}$ & $\begin{array}{l}\text { Economía 4to } \\
\text { Año }\end{array}$ & $\begin{array}{l}\text { Economía 4to } \\
\text { año Formación } \\
\text { Orientada }\end{array}$ & $\begin{array}{l}\text { Economía 4to } \\
\text { año Formación } \\
\text { Orientada }\end{array}$ & $\begin{array}{l}\text { Economía (CENS- } \\
\text { Nocturno) }\end{array}$ \\
\hline \multicolumn{2}{|c|}{ Tipo de gestión } & Gestión privada & Escuela UBA & Gestión privada & Gestión Pública & Gestión pública \\
\hline \multicolumn{2}{|c|}{ Turno } & Mañana & Mañana & Mañana & Vespertina & Nocturna \\
\hline \multirow[t]{2}{*}{ 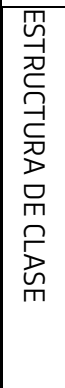 } & Agrupamiento & Todo el curso & Todo el curso & Todo el curso & $\begin{array}{l}\text { Formal: todo el } \\
\text { curso } \\
\text { Informal: } \\
\text { personal }\end{array}$ & $\begin{array}{l}\text { Se diferencian } \\
\text { dos grupos según } \\
\text { la conexión a } \\
\text { internet y/o } \\
\text { disponibilidad de } \\
\text { dispositivo } \\
\end{array}$ \\
\hline & Espacios & Aula virtual & Aula virtual & Aula virtual & $\begin{array}{l}\text { Aula virtual } \\
\text { fallida }\end{array}$ & $\begin{array}{l}\text { Aula virtual } \\
\text { fallida }\end{array}$ \\
\hline
\end{tabular}




\begin{tabular}{|c|c|c|c|c|c|}
\hline Tiempo & $\begin{array}{l}\text { Clase sincrónica } \\
40 \text { minutos }\end{array}$ & $\begin{array}{l}\text { Clase sincrónica } \\
40 \text { a } 60 \text { minutos }\end{array}$ & $\begin{array}{l}\text { Clase sincrónica, } \\
\text { se graba y queda } \\
\text { disponible para } \\
\text { estudiantes } \\
40 \text { minutos }\end{array}$ & $\begin{array}{l}\text { Clase sincrónica } \\
\text { por área } \\
\text { Sincronía a } \\
\text { demanda de } \\
\text { estudiantes }\end{array}$ & $\begin{array}{l}\text { Clase sincrónica } \\
\text { (60 minutos) } \\
\text { Asincrónicas }\end{array}$ \\
\hline Frecuencia & Semanal & Semanal & $\begin{array}{l}\text { Dos veces por } \\
\text { semana }\end{array}$ & $\begin{array}{l}\text { Semanal } \\
\text { A demanda de } \\
\text { estudiantes }\end{array}$ & $\begin{array}{l}\text { Semanal } \\
\text { No definido }\end{array}$ \\
\hline Comunicación & $\begin{array}{l}\text { Plataforma } \\
\text { reuniones } \\
\text { virtuales } \\
\text { Correo } \\
\text { electrónico con } \\
\text { estudiantes }\end{array}$ & $\begin{array}{l}\text { Plataforma } \\
\text { reuniones } \\
\text { virtuales } \\
\text { Correo } \\
\text { electrónico con } \\
\text { estudiantes }\end{array}$ & $\begin{array}{l}\text { Plataforma } \\
\text { reuniones } \\
\text { virtuales } \\
\text { Correo } \\
\text { electrónico con } \\
\text { estudiantes }\end{array}$ & $\begin{array}{l}\text { Mensajería por } \\
\text { celular }\end{array}$ & $\begin{array}{l}\text { Plataforma de } \\
\text { reuniones } \\
\text { virtuales } \\
\text { Correo } \\
\text { electrónico. } \\
\text { Mensajería por } \\
\text { celular. }\end{array}$ \\
\hline
\end{tabular}

\section{Bibliografía}

Arata, N. (2020). La escuela frente a la pandemia. Entre la defensa de lo común y la búsqueda de alternativas. En I. Dussel, P. Ferrante y D. Pulfer, Pensar la educación en tiempos de pandemia. Entre la emergencia, el compromiso y la espera (pp. 53-62). Editorial Universitaria.

Barberis, E. y Toibero, D. (2020). Sostener lo escolar en tiempos de emergencia. Re-construcción de una experiencia. En L. Beltramino, Aprendizajes y prácticas educativas en las actuales condiciones de época: COVID-19 (pp. 200-206). Universidad Nacional de Córdoba. Facultad de Filosofía y Humanidades. Basabe, L. y Cols, E. (2010). La enseñanza. En A. W de Camilloni, E. Cols, L. Basabe y S. Feeney, El saber didáctico (pp. 125-161). Paidós.

Cols, E. (2007). Sentidos personales y configuraciones de acción tras la semejanza de las palabras [Tesis de doctorado]. Facultad de Filosofía y Letras. Universidad de Buenos Aires.

Contreras, J. (1990). Enseñanza, currículum y profesorado. Ediciones Akal.

Davini, M. (2015). La didáctica y la práctica docente. Paidos.

Dussel, I. (2020). La clase en pantuflas. En I. Dussel, P. Ferrante y D. Pulfer, Pensar la educación en tiempos de pandemia : entre la emergencia, el compromiso y la espera (pp. 337-350). Editorial Universitaria.

Glaser, B. y Strauss, A. (1967). The discovery of grounded theory. Aldine Publishing Company. Jackson, P. (1975). La vida en las aulas. Marova.

Ministerio de Educación de la Nación. (2020). Informe preliminar: encuesta a docentes. Ministerio de Educación de la Nación.

Roldán, P. (2020). Dar clase con medios digitales. En L. Beltramino, Aprendizajes y prácticas educativas en las actuales condiciones de época: COVID-19 (pp. 26-31). Universidad Nacional de Córdoba. Facultad de Filosofía y Humanidades.

Sadovsky, P. y Castorina, J. (2020). Enseñar en tiempos de excepción: nuevos desafíos pedagógicos, incertidumbre y reconocimiento social. En I. Dussel, P. Ferrante y D. Pulfer, Pensar la educación en tiempos de pandemia II. Experiencias y problemáticas en Iberoamérica (pp. 211-224). Editorial Universitaria-CLACSO. Soneira, A. (2006). La «Teoría fundamentada en los datos» (Grounded Theory) de Glaser y Strauss. En I. Vasilachis de Gialdino, Estrategias de investigación cualitativa (pp. 153-173). Gedisa. 
Terigi, F. (2020). Aprendizaje en el hogar comandado por la escuela: cuestiones de descontextualización y sentido. En I. Dussel, P. Ferrante y D. Pulfer, Pensar la educación en tiempos de pandemia : entre la emergencia, el compromiso y la espera (pp. 243-250). Editorial Universitaria.

i La tesis busca aportar evidencias respecto al "cómo" se enseña Economía en la escuela secundaria. Para ello, se describen las prácticas de enseñanza de docentes de economía en escuelas secundarias de la Ciudad de Buenos Aires en el contexto institucional, áulico y para un grupo de estudiantes determinado. La elección de la disciplina se corresponde con un interés del autor que se desempeña como docente de Economía en las escuelas secundarias. En tanto, la delimitación a la Ciudad de Buenos Aires se explica porque la jurisdicción tiene un espacio curricular obligatorio para Economía en el tercer año de todas las escuelas secundarias, no es el caso de otras jurisdicciones, por ejemplo la Provincia de Buenos Aires, que solo tiene la materia Economía en el bachillerato orientado en Economía y Administración.

"Este elemento aparece con fuerza en el Informe preliminar de la encuesta a docentes del Ministerio de Educación de la Nación (2020), donde el criterio más importante para diseñar las propuestas de continuidad pedagógica en el nivel secundario fueron las pautas de los equipos de conducción.

iii En el Informe preliminar de la encuesta a docentes del Ministerio de Educación de la Nación (2020), el uso de mensajes de texto por teléfono celular es el medio de comunicación más utilizado junto con el correo electrónico.

iv En el Cuadro 1, se puede encontrar una sistematización de las variables consideradas.

${ }^{\vee}$ Conviene referenciar nuevamente el Informe preliminar de la encuesta a docentes del Ministerio de Educación de la Nación (2020): afirma que la frecuencia en la comunicación de docentes y estudiantes es, en el $65 \%$ de los casos, de una o más veces por semana. 